\title{
Inverse Slip Accompanying Twinning and Detwinning during Cyclic Loading of Magnesium Single Crystal
}

\author{
Qin Yu, ${ }^{1,2}$ Jian Wang, ${ }^{2}$ and Yanyao Jiang' \\ ${ }^{1}$ Department of Mechanical Engineering, University of Nevada, Reno, NV 89557, USA \\ ${ }^{2}$ Materials Science and Technology Division, Los Alamos National Laboratory, Los Alamos, NM 87545, USA \\ Correspondence should be addressed to Jian Wang; wangj6@lanl.gov and Yanyao Jiang; yjiang@unr.edu
}

Received 5 May 2013; Revised 18 July 2013; Accepted 19 July 2013

Academic Editor: Xiaozhou Liao

Copyright (c) 2013 Qin Yu et al. This is an open access article distributed under the Creative Commons Attribution License, which permits unrestricted use, distribution, and reproduction in any medium, provided the original work is properly cited.

In situ, observation of twinning and detwinning in magnesium single crystals during tension-compression cyclic loading was made using optical microscopy. A quantitative analysis of plastic strain indicates that twinning and detwinning experience two stages, low and high work hardening de-twinning, and pure re-twinning and fresh twinning combined with retwinning. Slip is always activated. For the first time, inverse slip accompanying with pure retwinning and high work hardening detwinning was experimentally identified, which provides insights in better understanding of the activity of twining, detwinning, and slips.

\section{Introduction}

Plastic deformation in materials with hexagonal-closepacked (HCP) structure is accommodated by both slip and twinning (or detwinning) $[1,2]$. For magnesium $(\mathrm{Mg})$ alloys, twinning is mainly related to the extension twin $\{10 \overline{1} 2\}\langle 10 \overline{1} 1\rangle$ with six variants that can be activated by an equivalent tension along the $c$-axis. Due to its polar nature, detwinning leads to the reorientation of the basal pole from the twin back to the matrix [1-4]. Under cyclic loading and strain path changes, twinning and detwinning appear alternately [59]. Experimental studies have been focusing on the role of twinning and detwinning in cyclic deformation and fatigue [5-9]. In addition to twinning, slips are necessary to accommodate arbitrary plastic deformation. However, the activity of twinning and slip is different in terms of nucleation and glide (or growth). For instance, nucleation of lattice glide dislocations is relatively easier than twins, but the mobility of a $\{10 \overline{1} 2\}$ twinning dislocation is greater than that of a lattice dislocation [10-14]. As a consequence, the plastic deformation in the twinned crystal resulted from twinning shear can be greater than that in the matrix resulted from dislocation slip. The incompatibility of plastic deformation between the twin and the matrix can generate a change in the stress state in the matrix surrounding the growing twin. To be specific, the shear stress in the matrix near the growing twin can have an opposite sign to the shear stress in the matrix far away from the twin. The opposite signed stress surrounding the twin is often referred to as "backstress." The growth of twin can be slowed down and even stopped if the local backstress keeps increasing. To sustain the growth of twin, the magnitude of the backstress must be reduced or the direction of the backstress should reorient to align with that of the external load. Such changes can be achieved through either increasing the external load or initiating slips in the direction opposite to the twinning shear (or the external load). Note that at any moment, the total plastic strain rate $\left(\dot{\varepsilon}_{P(i j)}^{\text {Exp }}\right)$ with respect to time is equal to the summation of the twinning-induced plastic strain rate $\left(\dot{\varepsilon}_{P(i j)}^{\mathrm{Tw}}\right)$ and the slipinduced plastic strain rate $\left(\dot{\varepsilon}_{P(i j)}^{S}\right)$; that is, $\dot{\varepsilon}_{P(i j)}^{\operatorname{Exp}}=\dot{\varepsilon}_{P(i j)}^{\mathrm{Tw}}+\dot{\varepsilon}_{P(i j)}^{S}$, where the subscripts $i$ and $j$ denote the coordinates. If a slip flows in the direction opposite to that of the twinning shear, we define such a slip as "inverse slip." To validate the existence of inverse slips, it is essential to experimentally and quantitatively separate the plastic deformation contributed by twinning/detwinning and slips.

In the current study, a tension-compression cyclic loading experiment of a [0001]-orientated $\mathrm{Mg}$ single crystal was conducted at room temperature. In situ, optical microscopy 
was employed to directly observe the evolution of twins. Optical microscopy (OM) was chosen for the study due to the large observation area and high frame rate [5]. A large observation area is extremely crucial for the determination of macrostrain that is equal to the strain measured by using an extensometer in the tension-compression experiment of a single crystal. In addition, twins are generally larger than micrometers in size and grow very fast. Therefore, an optical microscope equipped with a fast digital camera can properly capture the twin growth. To this end, in situ high/low resolution transmission electron microscopy (TEM) or scanning electron microscope or electron backscatter diffraction is not suitable to study the cyclic tension-compression deformation due to the geometry limit of the testing samples and due to the small observation region and the low frame rate [15-19]. For example, the buckling could occur for a TEM sample that is subjected to a cyclic loading with a total strain magnitude of $0.5 \%$.

\section{Material, Experiment, and Analysis Method}

$\mathrm{Mg}$ single crystal was grown by the Bridgman method [20]. Small plate dog-bone testing specimen is cut by chemical corrosion technique [5] and has a gage length of $5.0 \mathrm{~mm}$ and a square cross-section of $3.0 \mathrm{~mm}$ by $3.0 \mathrm{~mm}$. The loading direction was aligned with the $c$-axis. The specimen surface, that is, the $(10 \overline{1} 0)$ prismatic plane, was carefully polished down to $1 \mu \mathrm{m}$ using polycrystalline diamond suspension, followed by chemical polishing using a solution of 1 part of nitric acid, 2 parts of hydrochloric acid, and 7 parts of ethanol as described in [21]. The well-prepared specimen was characterized using OM, and no mechanical twins were detected before the testing. A servohydraulic load frame with a load capacity of $\pm 25 \mathrm{kN}$ was employed to perform the cyclic loading experiment. A grip system was specially designed to ensure the accurate alignment of the loading axis with the testing specimen. A strain-gaged extensometer with a range of $\pm 9.15 \%$ was attached to the specimen to directly measure the engineering strain within the gage length of the testing sample. The load was measured by the load cell and recorded by a computer. A long-distance optical microscope was employed to trace the twins in the middle of the specimen observation surface during testing. The image frame covers an area of $1.4 \mathrm{~mm} \times 1.0 \mathrm{~mm}$ on the specimen surface with a resolution of approximate $1 \mu \mathrm{m}$ per pixel. The video recording rate was 42 frames per minute. Fully reversed strain-controlled tension-compression was applied at a total strain amplitude of $0.5 \%$ at a frequency of $0.01 \mathrm{~Hz}$ in ambient air.

The plastic strain, $\varepsilon_{p}(t)$, is determined by subtracting the elastic strain, $\varepsilon_{e}(t)$, from the total applied strain $\varepsilon^{\operatorname{Exp}}(t)$. The elastic strain $\varepsilon_{e}(t)$ is calculated using the Hooke's Law employing a constant Young's modulus of $44.7 \mathrm{GPa}$. The plastic strain consists of two parts: twinning-induced and slip-induced plastic strains. The twinning-induced plastic strain, $\varepsilon_{p}^{\mathrm{Tw}}(t)$, is determined according to the measured twin volume fraction that is identified in optical micrographs through an image analysis. With the given crystallography and the observation direction under investigation, there are six $\{10 \overline{1} 2\}$ extension twin variants that could be equally activated due to identical Schmid factors of the variants (Figure 1(a)). For each twin variant, the twin volume fraction is calculated from the twin area fraction by multiplying a geometrical factor $\beta_{i}(i=1,2$, $3, \ldots, 6$, denoting the six variants) that accounts for the true twin thickness. $\beta$ is equal to 0.73 for two variants and 0.94 for the other four variants. Due to a technique difficulty to numerically distinguish the type of twin variants, an average geometry factor $\bar{\beta}=(1 / 6) \sum_{i=1}^{6} \beta_{i}$ was adopted in this study (to avoid an overestimation of the contribution to the plastic deformation by twins, we also performed an analysis by using a low geometry factor of 0.73 , in Figure S1. The results from both low and averaged geometry factors are similar). The total twin area fraction $A$ is equal to the ratio of the total number of dark contrasted pixels (representing twins) over the total number of pixels within the observed region. The plastic strain accommodated by twinning/detwinning is calculated according to the volume average plastic strain [22], $\varepsilon_{p(i j)}^{\mathrm{Tw}}=\left(\gamma_{t w} \bar{\beta} A / 2\right)(m \otimes n+n \otimes m)$, where $\varepsilon_{p(i j)}^{\mathrm{Tw}}$ is the plastic strain accommodated by twinning, $\gamma_{t w}$ is the twinning shear defined as $\sqrt{3} /(c / a)-(c / a) / \sqrt{3}$, which is equal to 0.129 as $c / a=1.624$ for $\mathrm{Mg}$, and $m$ and $n$ are the normal vectors of the twin plane and the twinning direction corresponding to the specimen's coordinates, respectively. Slip-induced strain is the difference between the measured plastic strain and the twinning-induced plastic strain, that is, $\varepsilon_{p(i j)}^{S}=\varepsilon_{p(i j)}^{\operatorname{Exp}}-\varepsilon_{p(i j)}^{\mathrm{Tw}}$. The subscripts $i$ and $j$ represent the specimen coordinates $x, y$, and $z$. Since the experiment was conducted with the controlled strain along the $z$-axis (see Figure 1), only the strain components in the $z$ direction $\left(\varepsilon_{p(z z)}^{\operatorname{Exp}}, \varepsilon_{p(z z)}^{\text {Tw }}\right.$, and $\left.\varepsilon_{p(z z)}^{S}\right)$ are analyzed and discussed. A parabolic curve is used to fit a successive set of five data points in the experimentally obtained relationship between the plastic strain $\left(\varepsilon_{p(z z)}^{\operatorname{Exp}}, \varepsilon_{p(z z)}^{\mathrm{Tw}}\right.$, or $\left.\varepsilon_{p(z z)}^{S}\right)$ and the time. The first-order derivative of the obtained fitting curve at the middle point in the successive five-point data set is reported as the plastic strain rates $\left(\dot{\varepsilon}_{p(z z)}^{\operatorname{Exp}}\right.$, $\dot{\varepsilon}_{p(z z)}^{\mathrm{Tw}}$, and $\left.\dot{\varepsilon}_{p(z z)}^{S}\right)$.

With the increasing number of loading cycles after the tenth loading cycle, residual twins are found to accumulate significantly. Large amounts of crossing-like twin-twin junctions are developed severely at local areas, for example, as shown in the optical micrographs at the tensile peak strain and compressive peak strain in the 40th, 400th, and 1610th loading cycle in Figure 2. To ensure the reliability of the analysis, we only report the plastic strains (total, twinning induced, and slip induced) during the first ten loading cycles, where much fewer crossing-like twin-twin junctions were observed (see Figure 1 and Supplementary Material MOVIE available online at http://dx.doi.org/10.1155/2013/903786).

\section{Results and Discussion}

Figure 3(a) illustrates a typical stress-plastic strain hysteresis loop for the $i$ th loading cycle with four featured segments divided by critical points from $A_{i}, B_{i}, C_{i}, D_{i}, E_{i}$, and $A_{i+1}$. These four featured segments are related to slips and three 


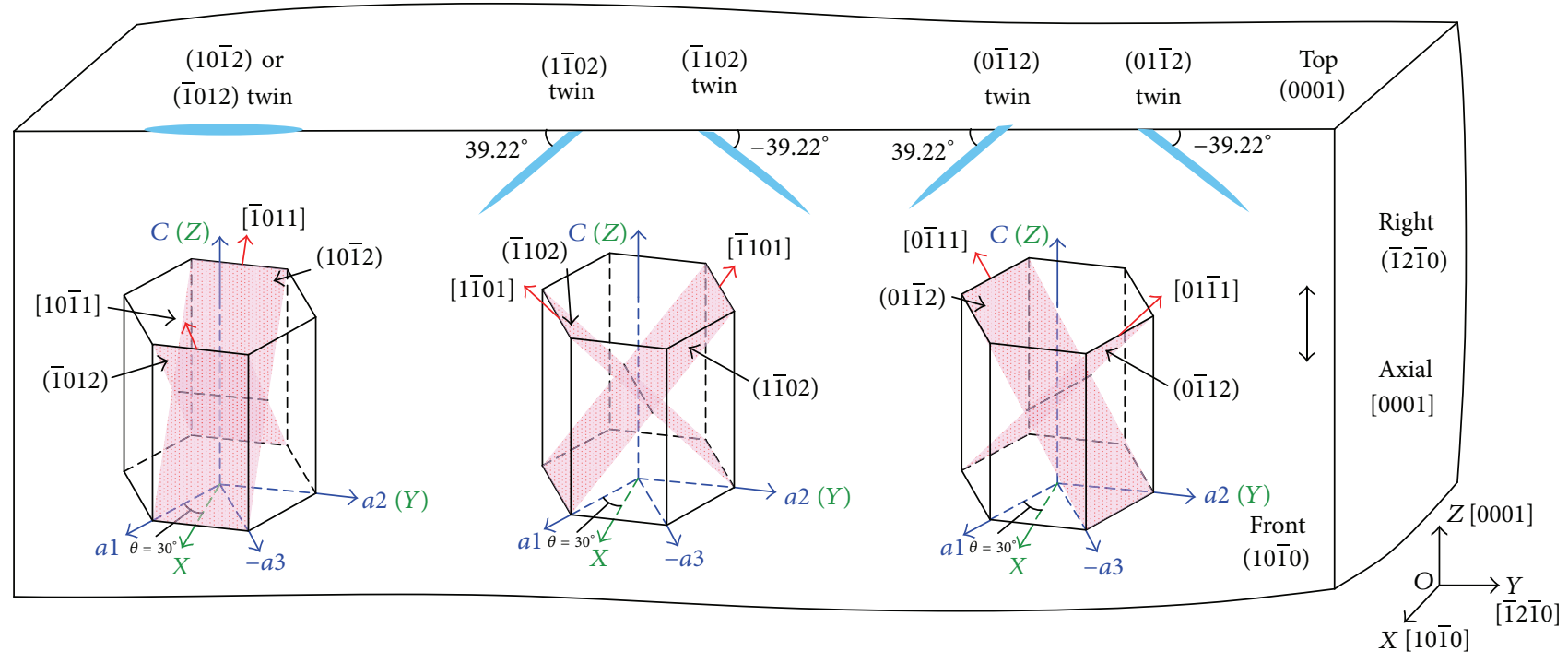

(a)

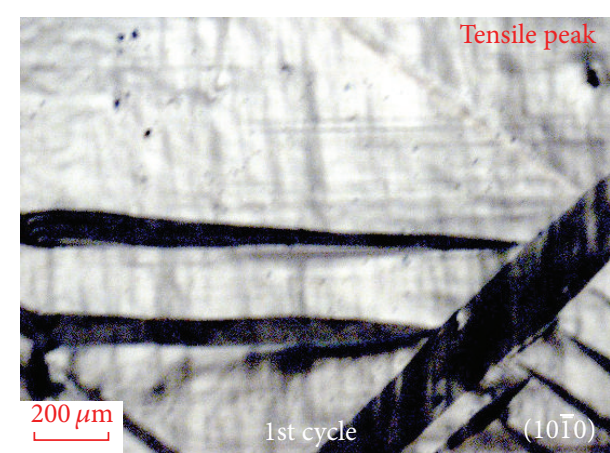

(b)

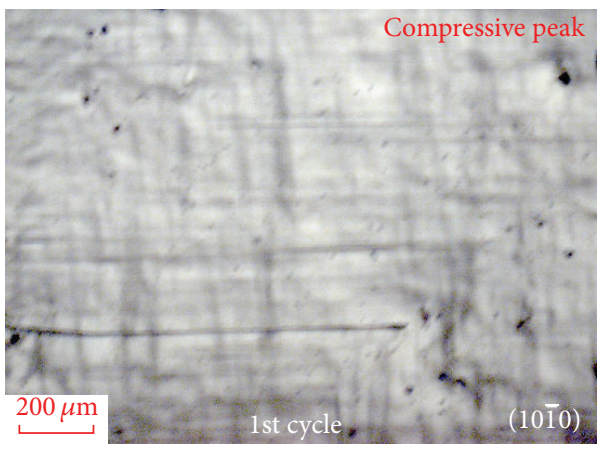

(d)

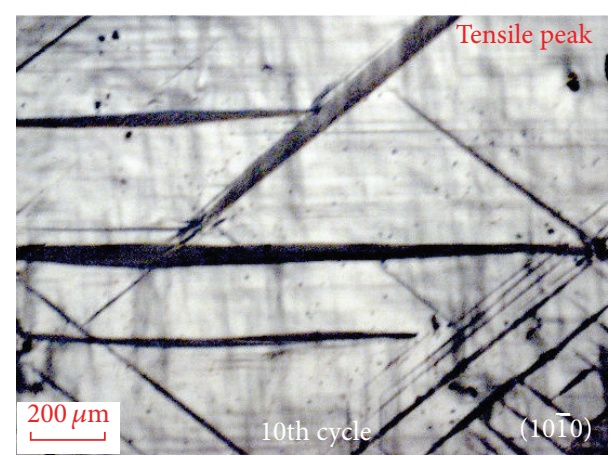

(c)

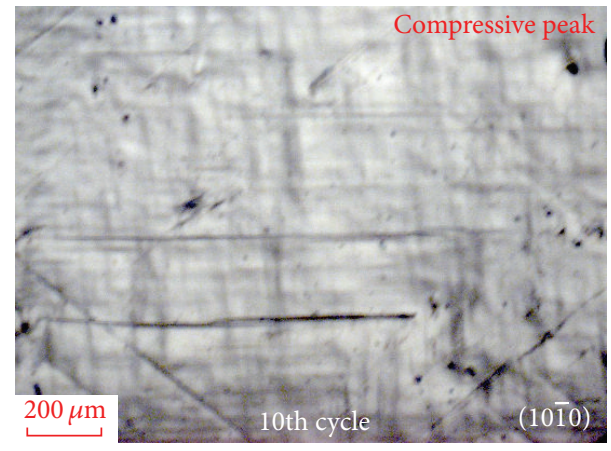

(e)

Figure 1: (a) Schematics showing six $\{10 \overline{1} 2\}$ twin variants and their projections on the (10)̄0) prismatic plane. In situ optical micrographs showing twins at the tensile peak strain ((b) and (c)) and compressive peak strain ((d) and (e)) at the first loading cycle and the tenth loading cycle.

distinctive twinning mechanisms: fresh twinning, detwinning, and retwinning. Fresh twinning refers to the nucleation and propagation of new twins from the virgin, material and retwinning corresponds to the repeated growth of a residual twin that is not fully detwinned [5]. $A_{i}$ and $C_{i}$ are defined as points at the tensile and compressive peak strain, respectively. Stage from $A_{i}$ and $C_{i}$ refers to the unloading including the tensile unloading and the compressive reloading. We introduced a critical point $B_{i}$, from $A_{i}$ to $B_{i}$, and plastic deformation is mainly accommodated by the easy detwinning (corresponding to a low work hardening). From $B_{i}$ to $C_{i}$, detwinning and slips both accommodate plastic deformation, causing a high work hardening. Due to the dislocation-twin interaction, detwinning becomes difficult, thus referred to 


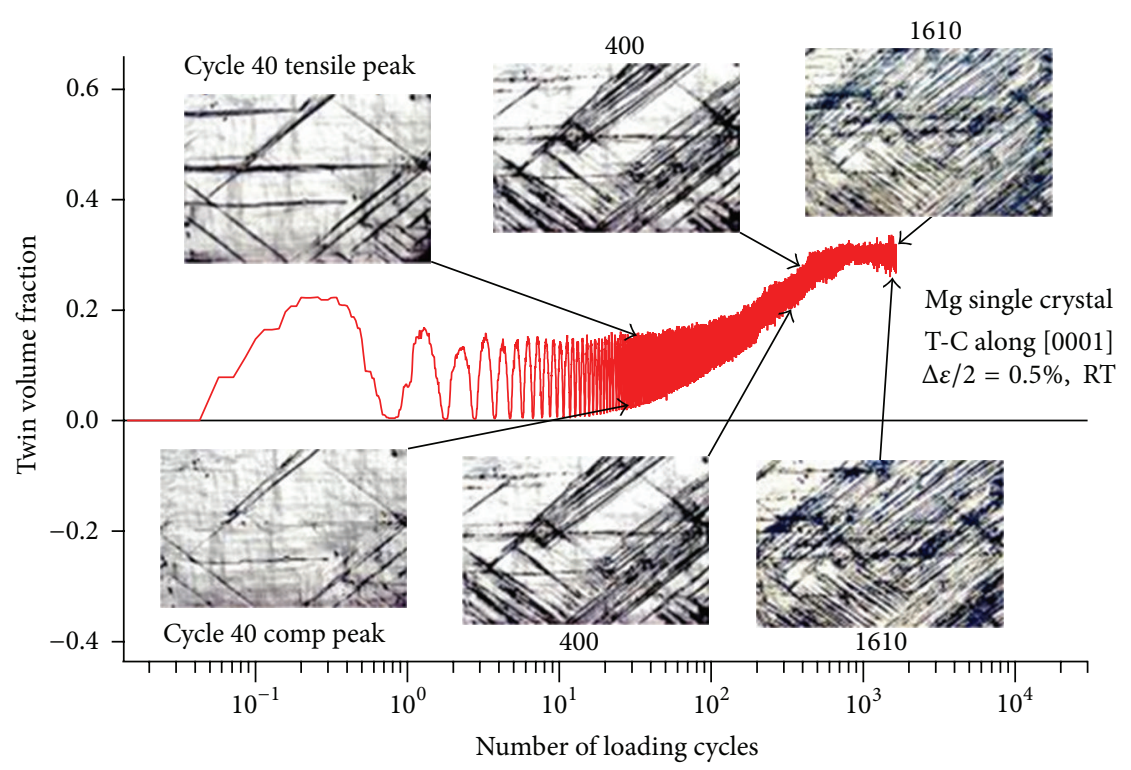

FIGURE 2: Variation of twin volume fraction with respect to the loading cycles together with the optical micrographs showing twins at tensile and compressive peak strains in the 40th, 400th, and 1610th loading cycle.

as the high work hardening detwinning. The critical point $B_{i}$ is defined as the point where the plastic strain rate induced by slip inverses its sign from negative to positive in Figure 3(c). The critical point $D_{i}$ is defined as the end of the elastic segment following the compressive strain peak $C_{i}$ in Figure 3(d), during which no plastic flow exists. Similar to the case of $B_{i}$, the transition point $E_{i}$ from pure retwinning to reand fresh-twinning is defined as the point at which the slipinduced plastic strain rate inverses its sign from negative to positive in Figure 3(c).

Figure 4(a) presents the stress-plastic strain hysteresis loops for the first and second loading cycles. Six snapshots from in situ optical micrographs show morphology change of twins at critical points $\left(A_{1}, B_{1}, C_{1}, D_{1}, E_{1}\right.$, and $\left.A_{2}\right)$. The variations of plastic strain, plastic strain rates, and the absolute activities of twinning and slip with respect to the time are also quantified, as shown in Figure 4(b)-Figure 4(e). The activity of twin or slip is a normalized factor that is used to indicate the percentage of plastic deformation in association with twinning or slip during a deformation segment. For example, $f_{\mathrm{Tw}}^{A_{i} B_{i}}=\left(\Delta \varepsilon_{p(z z)}^{\mathrm{Tw}}\right)_{A_{i} B_{i}} /\left(\Delta \varepsilon_{p(z z)}^{\mathrm{Tw}}+\Delta \varepsilon_{p(z z)}^{S}\right)_{A_{i} B_{i}}$ is for twin activity during deformation from $A_{i}$ to $B_{i}$, and $f_{S}^{A_{i} B_{i}}=$ $\left(\Delta \varepsilon_{p(z z)}^{\mathrm{Tw}}\right)_{A_{i} B_{i}} /\left(\Delta \varepsilon_{p(z z)}^{\mathrm{Tw}}+\Delta \varepsilon_{p(z z)}^{S}\right)_{A_{i} B_{i}}$ corresponds to the slip activity, where $\Delta$ denotes the plastic strain (twinning or slip) range between $A_{i}$ and $B_{i}$. In Figure $4(\mathrm{~d})$, the absolute value of the normalized activity factor is shown.

During initial tension (O to $\left.A_{1}\right)$, the six variants of $\{10 \overline{1} 2\}$ tensile twins are easily activated due to the same Schmid factor of 0.499 (see Figure 1). As observed in in situ optical microscopy (see Supplementary MOVIE), deformation twins nucleate instantaneously and grow very rapidly, forming fresh twins [5]. In Figures 4(c) and 4(d), it is noticed that the twininduced strain rate has the same sign as the applied total strain rate, but the slip-induced strain rate has an opposite sign once twinning is activated. At the tensile peak strain $\left(A_{1}\right)$, all the deformation twins are fully expanded (see micrograph $A_{1}$ in Figure 4(a)). From $A_{1}$ to $B_{1}$ in Figure 4(c), the slip-induced plastic strain rate is initially greater than the detwinninginduced plastic strain rate, but later approximately equals to zero, implying that detwinning mainly accommodates the applied strain. Comparing the optical micrographs $A_{1}$ and $B_{1}$ in Figure 4(a), detwinning of the fully expanded twins at $A_{1}$ is clearly observed. As revealed in Figures 4(d) and 4(e), both detwinning and slip-induced strain rates have the same sign as the applied strain rate. During tensile unloading and the early stage of compressive reloading, slip has a higher activity than detwinning (Figure 4(d)) and the magnitude of the stress quickly decreases, indicating recovery of slips (or reversible slip). Subsequently until point $B_{1}$, the stress-plastic strain curve shows slight strain hardening where detwinning has the higher activity than slip (Figure $4(\mathrm{~d})$ ). From $B_{1}$ to $C_{1}$ in Figure 4(c), detwinning-induced strain rate has the same sign as the applied strain rate, but slip-induced strain rate has an opposite sign, clearly showing the occurrence of inverse slip. Detwinning during this segment has a higher activity than the slip activity (Figure 4(d)). Differing from detwinning from $A_{1}$ to $B_{1}$, the magnitude of the stress quickly increases from $B_{1}$ to $C_{1}$. It is noted that $B_{1}$ in Figure 4 is a critical point corresponding to the transition from low work hardening detwinning to high work hardening detwinning, at which the plastic strain rate induced by slip inverses its sign from negative to positive (Figure $3(\mathrm{c})$ ). From $C_{1}$ to $D_{1}$, deformation is elastic, and $D_{1}$ corresponds to the yield point. No morphology change is observed by comparing optical micrographs at $C_{1}$ and $D_{1}$. From $D_{1}$ to $A_{2}$, retwinning occurs at a compressive stress of $-50 \mathrm{MPa}$ at the early stage $\left(D_{1}\right.$ to $\left.E_{1}\right)$ of reversed loading. As revealed in optical micrograph $E_{1}$, pure retwinning is terminated at $E_{1}$ with the appearance of two fresh twins (see Arrows $P_{1}$ and $P_{2}$ in Figure 4). During 


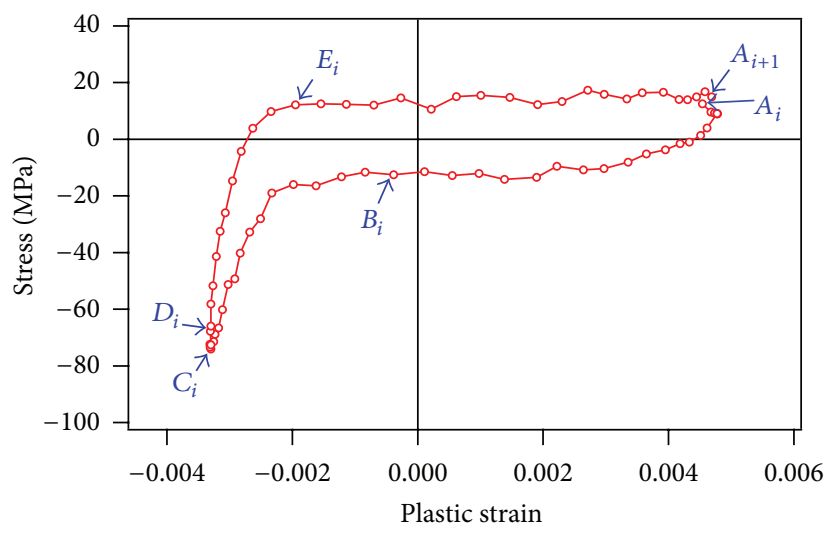

-O- Cycle $i\left(A_{i}\right.$ to $\left.A_{i+1}\right)$

$A_{i}$ to $B_{i}$ : detwinning

$B_{i}$ to $C_{i}$ : detwinning

(a)

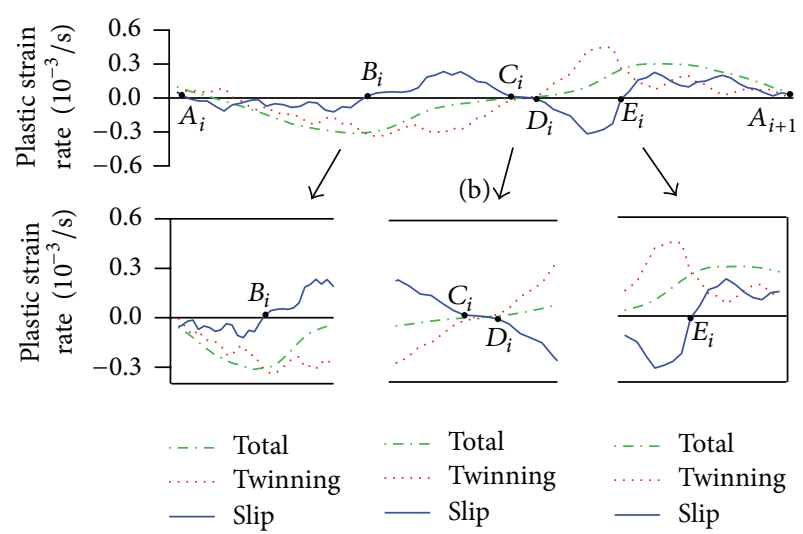

(c)

(e)

FIgURE 3: Stress-plastic strain hysteresis loop with critical points $\left(A_{i}\right.$ to $\left.A_{i+1}\right)$ for the $i$ th loading cycle (a) and variation of plastic strain rates (b) with the focused areas near critical point $B_{i}(\mathrm{c}), D_{i}(\mathrm{~d})$, and $E_{i}(\mathrm{e})$.

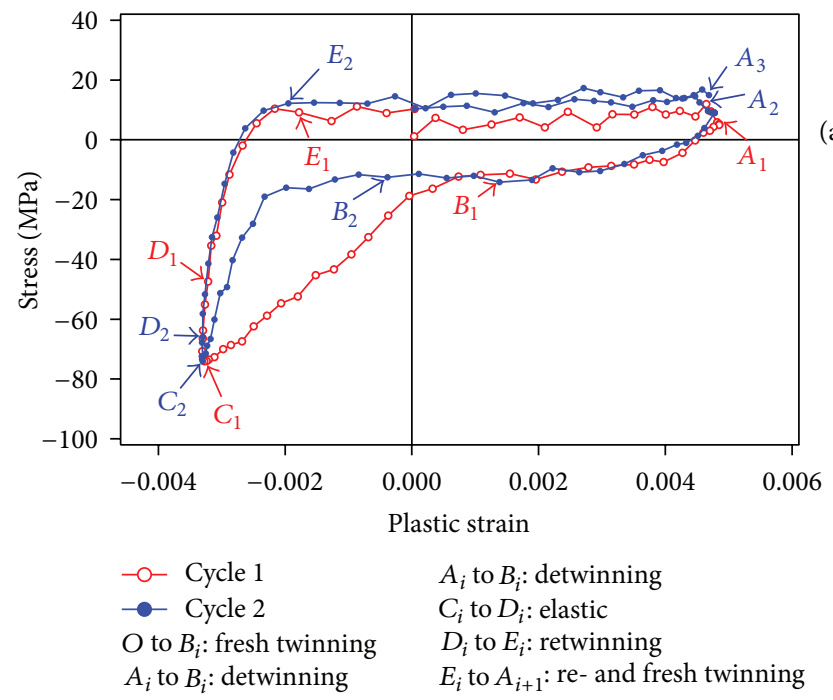

(a)
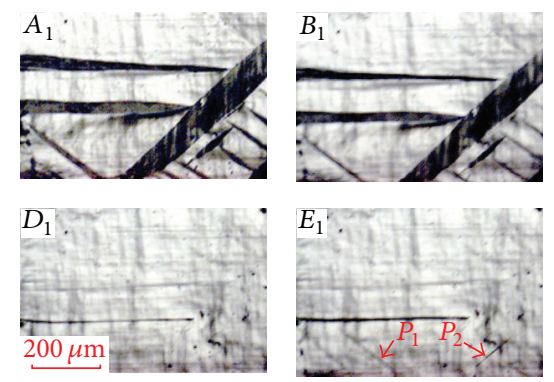
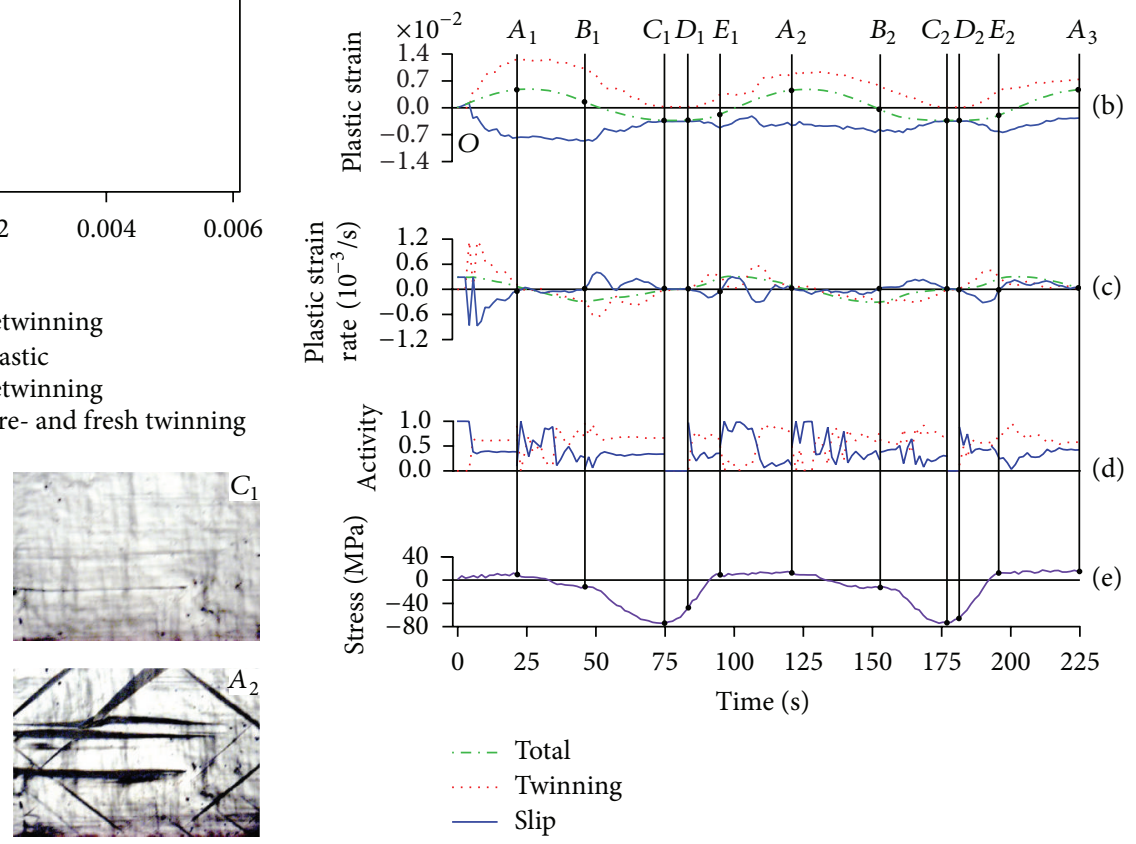

FIGURE 4: (a) Stress-plastic strain hysteresis loops at the critical points of the first and second loading cycles. In situ, optical micrographs at critical points $\left(A_{1}, B_{1}, C_{1}, D_{1}, E_{1}\right.$, and $\left.A_{2}\right)$ show the morphology change of twins. from (b) to (e) illustrate the variations of (b) plastic strains, (c) plastic strain rates, (d) absolute activity of twinning and slip which indicates the percentage of plastic deformation by twinning or slip during a deformation segment in the stress-plastic strain hysteresis loop, and (e) the stress with respect to the time for the first and second loading cycles. 


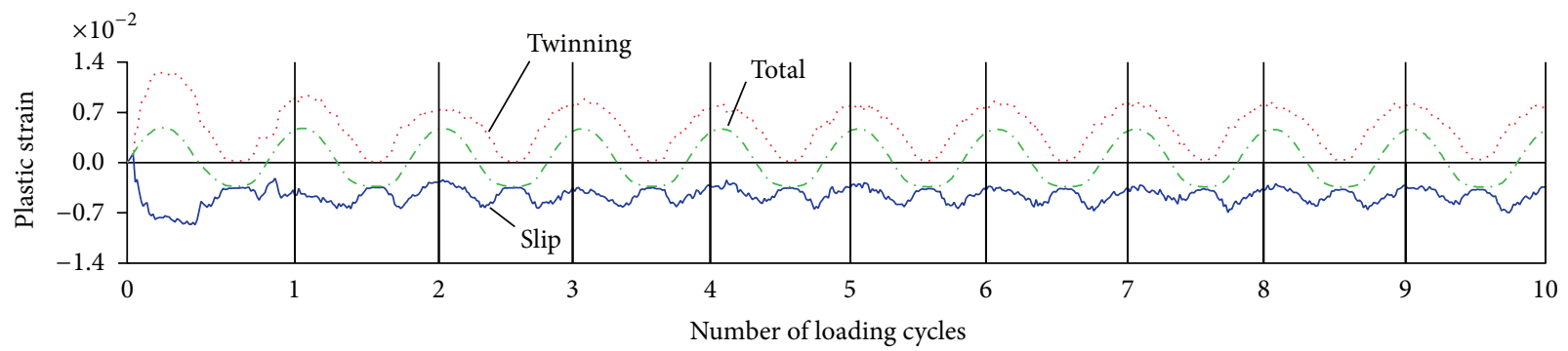

(a)

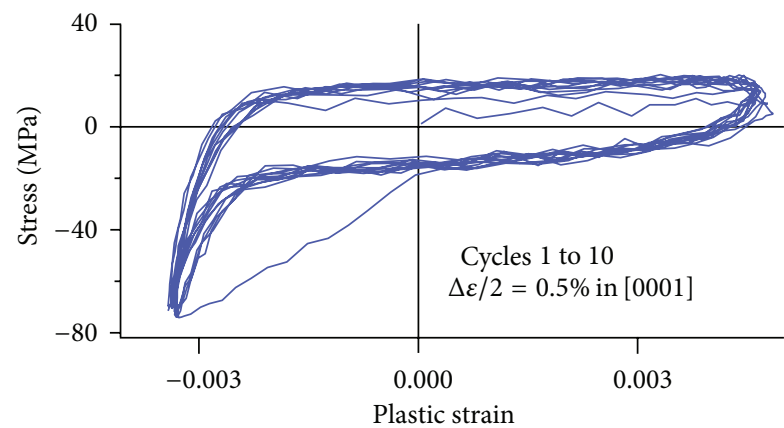

(b)

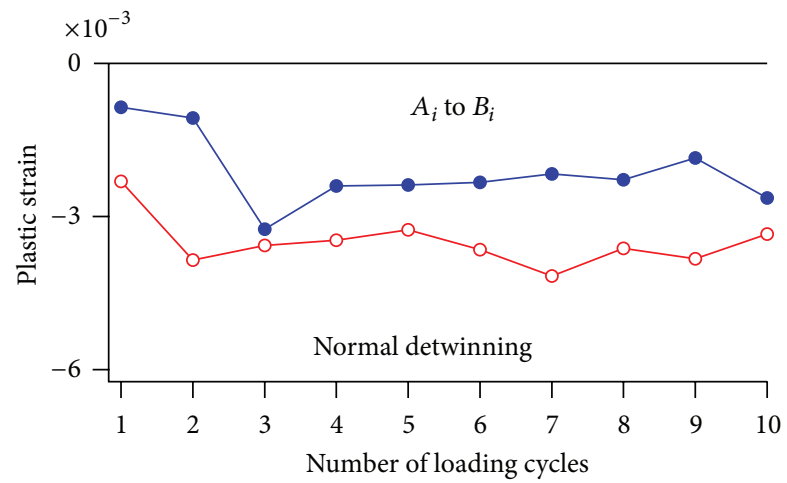

(d)

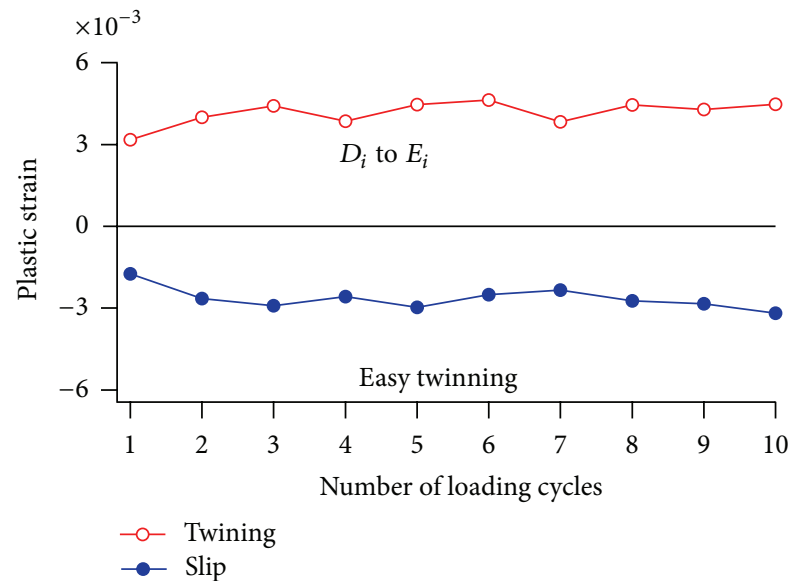

(f)

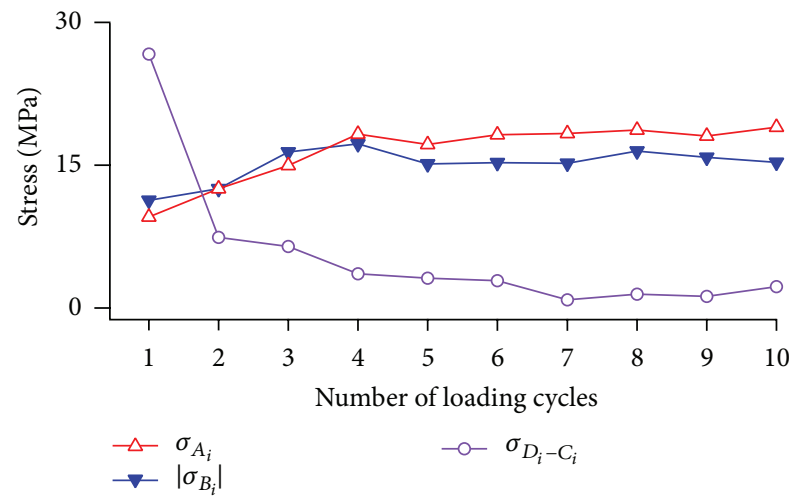

(c)

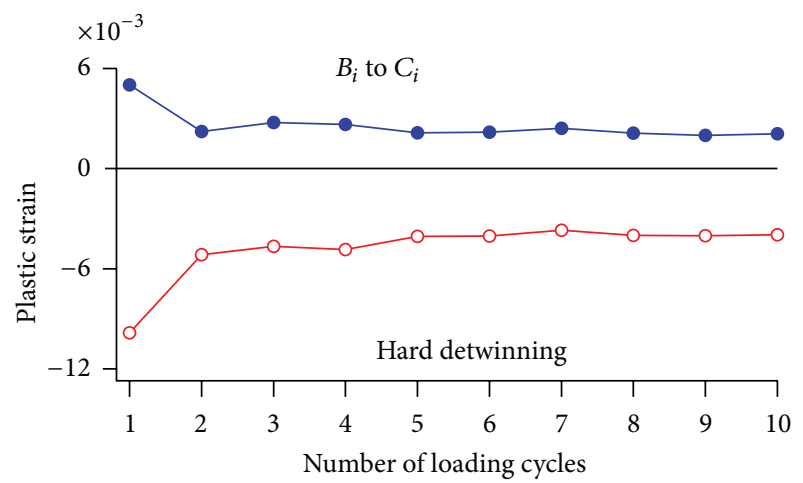

(e)

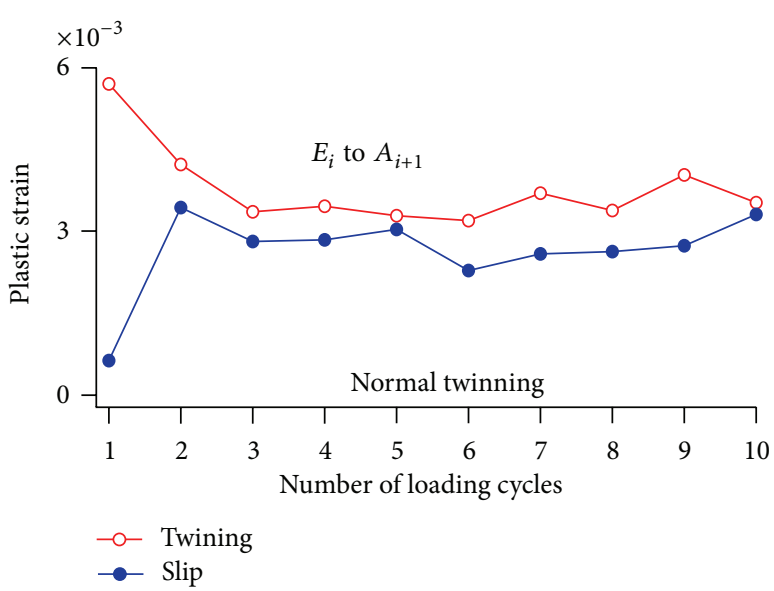

(g)

FIGURE 5: Dependence of plastic strains and other physical quantities associated with deformation mechanisms on the loading cycles. (a) Variations of twin-induced and slip-induced strains, (b) stress-plastic strain hysteresis loops, (c) the stresses at $A_{i}$ and $B_{i}$ and the stress range between $D_{i}$ and $C_{i}$. (d)-(g) Variation of twin-induced and slip-induced strains during (d) low work hardening detwinning, (e) high work hardening detwinning, (f) pure retwinning, and (g) retwinning and fresh twinning together. 
pure retwinning from $D_{1}$ to $E_{1}$, the slip-induced strain rate has an opposite sign to the applied plastic strain rate, indicating that inverse slip appears. After $E_{1}$, twinning during $E_{1}$ to $A_{2}$ is mainly associated with fresh twinning combined with retwinning, and the stress magnitude is approximately constant. As observed in optical micrograph $A_{2}$, several new twins are nucleated.

The second loading cycle $\left(A_{2}\right.$ to $\left.A_{3}\right)$ experiences a similar deformation process as the first loading cycle $\left(A_{1}\right.$ to $A_{2}$, see Figure 4). Deformation mechanism corresponding to the segment from $A_{2}$ to $B_{2}$ is the same as that from $A_{1}$ to $B_{1}$. The slip-induced plastic strain rate is initially greater than that associated with detwinning but later approaches approximately zero where detwinning mainly accommodates plastic deformation. From $B_{2}$ to $C_{2}$, detwinning-induced strain has the same sign as that of the applied strain, but slipinduced strain rate has an opposite sign. Deformation from $C_{2}$ to $D_{2}$ is elastic. From $D_{2}$ to $A_{3}$, the single crystal initially experiences re-twinning and subsequently fresh twinning. Inverse slip only accompanies with retwining and ends up at $E_{2}$, at which the sign of slip-induced rate changes from negative to positive.

Similar features were observed for the subsequent loading cycles (Figure 5) with typical four featured segments: (i) twinning from $D_{i-1}$ to $A_{i}$, including pure retwinning $\left(D_{i-1}\right.$ to $\left.E_{i-1}\right)$ and fresh twinning combined with retwinning $\left(E_{i-1}\right.$ to $A_{i}$ ), (ii) low work hardening detwinning from $A_{i}$ to $B_{i}$, (iii) high work hardening detwinning from $B_{i}$ to $C_{i}$, and (iv) elastic deformation from $C_{i}$ to $D_{i}$. To visualize the dependence of the deformation characteristics on loading cycle, Figure 5(a) plots the variations of twin-induced strain and slip-induced strain for the first ten loading cycles. A comprehensive analysis was performed for all loading cycles, and the results indicate that inverse slip always accompanies with pure retwinning $\left(D_{i}\right.$ to $\left.E_{i}\right)$ and high work hardening detwinning $\left(B_{i}\right.$ to $\left.C_{i}\right)$.

The variation of the stresses at $A_{i}$ and $B_{i}$ and the stress range between $D_{i}$ and $C_{i}$ are plotted in Figure 5(c) with respect to the loading cycles. The stress magnitude $B_{i}$ increases with loading cycles, which can be ascribed to strain hardening due to the dislocation-twin boundary interactions $[23,24]$. Accompanying with cyclic loading, more slips are activated and interact with the twin boundaries. Atomistic simulations combining with dislocation theory show that lattice glide dislocations can transmit across twin boundary or dissociate on twin boundary while the residual and sessile dislocations are left at twin boundary and further pin the twin boundaries [10, 23-26]. However, the elastic stress range between $D_{i}$ and $C_{i}$ decreases with increasing loading cycles, which could be mainly due to the accumulation of residual twins and the increase in the backstress resulted from detwinning. The peak stress at $A_{i}$ increases with loading cycles, indicating that strain hardening is the result of both dislocation-dislocation and dislocation-twin interactions [24]. The variation of twin activity and slip activity with respect to the four segments during cyclic loading are plotted in Figures 5(d)-5(g). The ratio of detwinninginduced strain over slip-induced strain is approximately 1.60 during low work hardening detwinning and is 1.20 during fresh twinning, while both accommodate the applied strain. However, during high work hardening detwinning and pure retwinning, the ratio is approximately -1.80 in average where inverse slips take place.

In summary, plastic deformation of [0001]-oriented $\mathrm{Mg}$ single crystals during cyclic loading can be featured with four segments that correspond to distinctive deformation mechanisms. Both slip and twin are always activated during plastic deformation, but twinning (or detwinning) is more active and contributes more to the total plastic strain. For the first time, inverse slip is found to be always accompanying with pure retwinning and high work hardening detwinning. This implies that twinning dislocation experiences a smaller Peierls stress than the lattice glide dislocation, which is in agreement with atomistic simulations [11]. In addition, retwinning can take place at macroscale compression accompanying with inverse slip, indicating that the local stress (which can be ascribed to the backstress resulted from the detwinning) has a significant effect on deformation mechanisms. The observed high work hardening detwinning associated with inverse slip and the increase of stress magnitude might be an indicator of strain hardening aroused from the dislocation-twin interaction. The current findings provide a new insight in understanding plastic deformation and will benefit the development of polycrystalline plastic models for hexagonal-close-packed crystals [27-29].

\section{Acknowledgments}

Jiang and Yu acknowledge support by the US Department of Energy, Office of Basic Energy Sciences under Grant no. DE-SC0002144. Wang and Yu gratefully acknowledge support from Office of Basic Energy Sciences, Project FWP 06SCPE401, under US DOE Contract no. W-7405-ENG-36. The authors acknowledge the valuable discussion with Dr. Carlos N Tome at Los Alamos National Laboratory and Professor Peidong Wu at McMaster University.

\section{References}

[1] J. W. Christian and S. Mahajan, "Deformation twinning," Progress in Materials Science, vol. 39, no. 1-2, pp. 1-157, 1995.

[2] J. Wang, I. J. Beyerlein, and C. N. Tomé, "An atomic and probabilistic perspective on twin nucleation in Mg," Scripta Materialia, vol. 63, no. 7, pp. 741-746, 2010.

[3] Y. N. Wang and J. C. Huang, "The role of twinning and untwinning in yielding behavior in hot-extruded $\mathrm{Mg}-\mathrm{Al}-\mathrm{Zn}$ alloy," Acta Materialia, vol. 55, no. 3, pp. 897-905, 2007.

[4] X. Y. Lou, M. Li, R. K. Boger, S. R. Agnew, and R. H. Wagoner, "Hardening evolution of AZ31B Mg sheet," International Journal of Plasticity, vol. 23, no. 1, pp. 44-86, 2007.

[5] Q. Yu, J. Zhang, and Y. Jiang, "Direct observation of twinningdetwinning-retwinning on magnesium single crystal subjected to strain-controlled cyclic tension-compression in $\left[\begin{array}{llll}0 & 0 & 0 & 1\end{array}\right]$ direction," Philosophical Magazine Letters, vol. 91, no. 12, pp. 757-765, 2011.

[6] S. M. Yin, H. J. Yang, S. X. Li, S. D. Wu, and F. Yang, "Cyclic deformation behavior of as-extruded Mg-3\%Al-1\%Zn," Scripta Materialia, vol. 58, no. 9, pp. 751-754, 2008. 
[7] S. Begum, D. L. Chen, S. Xu, and A. A. Luo, "Strain-controlled low-cycle fatigue properties of a newly developed extruded magnesium alloy," Metallurgical and Materials Transactions A, vol. 39, no. 12, pp. 3014-3026, 2008.

[8] L. Wu, A. Jain, D. W. Brown et al., "Twinning-detwinning behavior during the strain-controlled low-cycle fatigue testing of a wrought magnesium alloy, ZK60A," Acta Materialia, vol. 56, no. 4, pp. 688-695, 2008.

[9] J. Zhang, Q. Yu, Y. Jiang, and Q. Li, "An experimental study of cyclic deformation of extruded AZ61A magnesium alloy," International Journal of Plasticity, vol. 27, no. 5, pp. 768-787, 2011.

[10] A. Serra and D. J. Bacon, "A new model for $\{10 \overline{1} 2\}$ twin growth in hcp metals," Philosophical Magazine A, vol. 73, no. 2, pp. 333343, 1996.

[11] J. Wang, I. J. Beyerlein, J. P. Hirth, and C. N. Tomé, “Twinning dislocations on $\{\overline{1} 001\}$ and $\{\overline{1} 013\}$ planes in hexagonal closepacked crystals," Acta Materialia, vol. 59, no. 10, pp. 3990-4001, 2011.

[12] J. Wang, R. G. Hoagland, J. P. Hirth, L. Capolungo, I. J. Beyerlein, and C. N. Tomé, "Nucleation of a (over( $\left(\begin{array}{llll}\overline{1} & 0 & 1 & 2\end{array}\right)$ twin in hexagonal close-packed crystals," Scripta Materialia, vol. 61, no. 9, pp. 903-906, 2009.

[13] J. Wang, J. P. Hirth, and C. N. Tomé, “(over( $\left(\begin{array}{llll}1 & 0 & 1 & 2\end{array}\right)$ Twinning nucleation mechanisms in hexagonal-close-packed crystals," Acta Materialia, vol. 57, no. 18, pp. 5521-5530, 2009.

[14] B. Li and E. Ma, "Zonal dislocations mediating $\{10 \overline{1} 1\}\langle 10 \overline{12}\rangle$ twinning in magnesium," Acta Materialia, vol. 57, no. 6, pp. 1734-1743, 2009.

[15] J. R. Greer and J. T. M. de Hosson, "Plasticity in small-sized metallic systems: intrinsic versus extrinsic size effect," Progress in Materials Science, vol. 56, no. 6, pp. 654-724, 2011.

[16] A. M. Minor, S. A. Syed Asif, Z. Shan et al., "A new view of the onset of plasticity during the nanoindentation of aluminium," Nature Materials, vol. 5, no. 9, pp. 697-702, 2006.

[17] Z. Shan, E. A. Stach, J. M. K. Wiezorek, J. A. Knapp, D. M. Follstaedt, and S. X. Mao, "Grain boundary-mediated plasticity in nanocrystalline nickel," Science, vol. 305 , no. 5684, pp. 654657, 2004.

[18] L. Liu, J. Wang, S. K. Gong, and S. X. Mao, "High resolution transmission electron microscope observation of zero-strain deformation twinning mechanisms in Ag," Physical Review Letters, vol. 106, no. 17, Article ID 175504, 2011.

[19] Y. Yang, L. Wang, T. R. Bieler, P. Eisenlohr, and M. A. Crimp, "Quantitative atomic force microscopy characterization and crystal plasticity finite element modeling of heterogeneous deformation in commercial purity titanium," Metallurgical and Materials Transactions A, vol. 42, no. 3, pp. 636-644, 2011.

[20] P. W. Bridgman, "The compressibility of thirty metals as a function of pressure and temperature," Proceedings of the American Academy of Arts and Sciences, vol. 58, pp. 165-242, 1923.

[21] J.-T. Chou, H. Shimauchi, K.-I. Ikeda, F. Yoshida, and H. Nakashima, "Preparation of samples using a chemical etching for SEM/EBSP method in a pure magnesium polycrystal and analysis of its twin boundaries," Journal of Japan Institute of Light Metals, vol. 55, no. 3, pp. 131-136, 2005.

[22] P. M. Anderson, "Crystal-based plasticity," in Fundamentals of Metal Forming Analyses, chapter 8, John Wiley \& Sons, New York, NY, USA, 1995.

[23] D. Sarker and D. L. Chen, "Detwinning and strain hardening of an extruded magnesium alloy during compression," Scripta Materialia, vol. 67, pp. 165-168, 2012.
[24] M. R. Barnett, M. D. Nave, and A. Ghaderi, "Yield point elongation due to twinning in a magnesium alloy," Acta Materialia, vol. 60, no. 4, pp. 1433-1443, 2012.

[25] J. Wang and I. J. Beyerlein, "Atomic structures of symmetric tilt grain boundaries in hexagonal close packed (hcp) crystals," Modelling and Simulation in Materials Science and Engineering, vol. 20, no. 2, Article ID 024002, 2012.

[26] J. Wang, I. J. Beyerlein, and J. P. Hirth, "Nucleation of elementary $\{\overline{1} 011\}$ and $\{\overline{1} 013\}$ twinning dislocations at a twin boundary in hexagonal close-packed crystals," Modelling and Simulation in Materials Science and Engineering, vol. 20, no. 2, Article ID 024001, 2012.

[27] S. R. Agnew and Ö. Duygulu, "Plastic anisotropy and the role of non-basal slip in magnesium alloy AZ31B," International Journal of Plasticity, vol. 21, no. 6, pp. 1161-1193, 2005.

[28] H. Wang, B. Raeisinia, P. D. Wu, S. R. Agnew, and C. N. Tomé, "Evaluation of self-consistent polycrystal plasticity models for magnesium alloy AZ31B sheet," International Journal of Solids and Structures, vol. 47, no. 21, pp. 2905-2917, 2010.

[29] H. Wang, P. D. Wu, C. N. Tomé, and J. Wang, "A constitutive model of twinning and detwinning for hexagonal close packed polycrystals," Materials Science and Engineering A, vol. 555, pp. 93-98, 2012. 

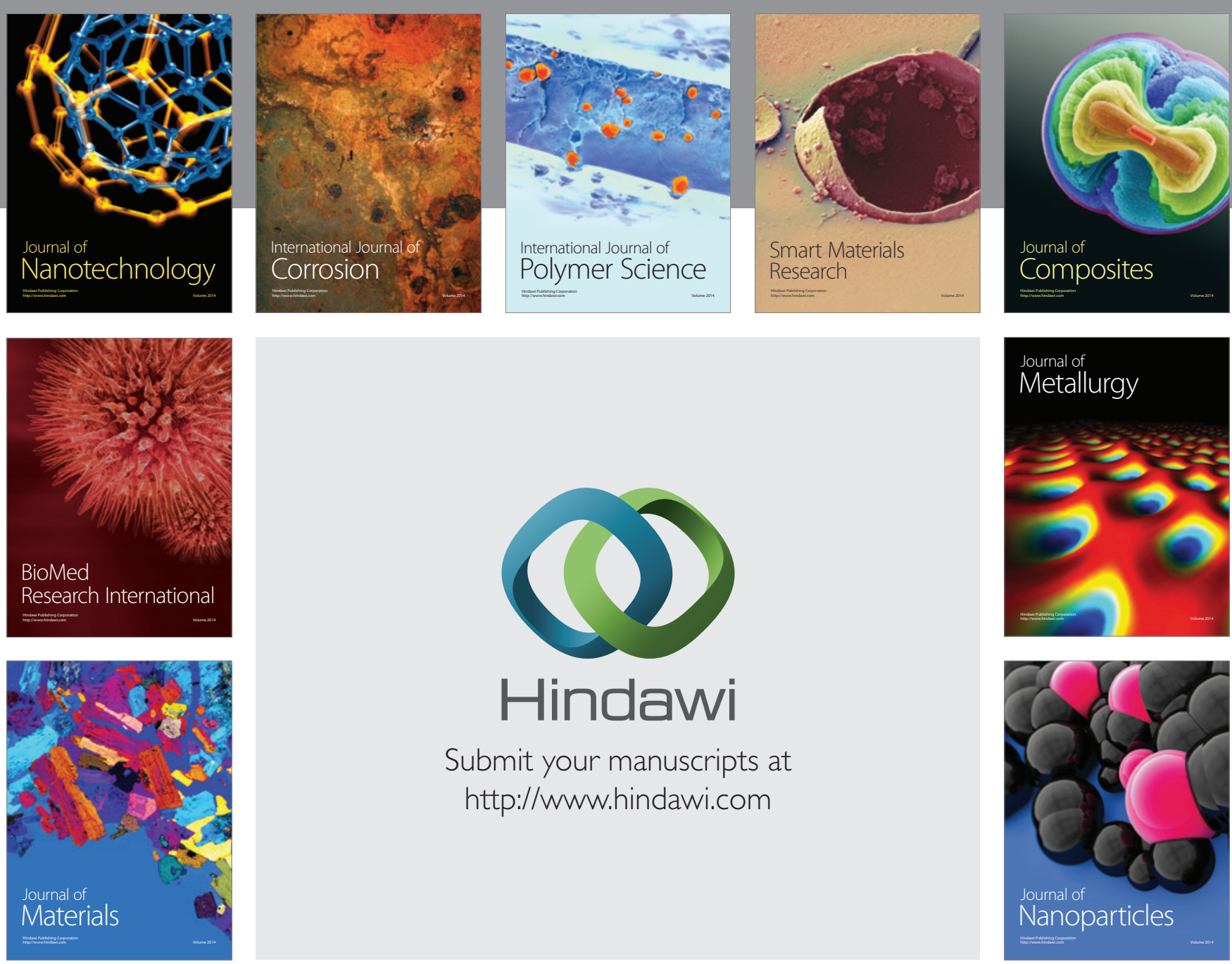

Submit your manuscripts at http://www.hindawi.com
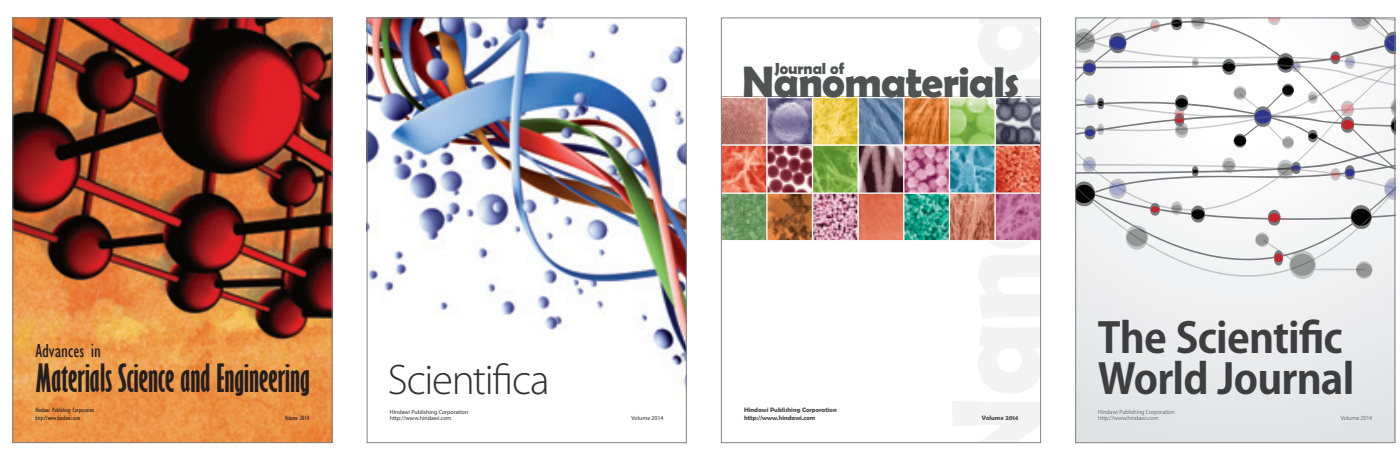

\section{The Scientific World Journal}
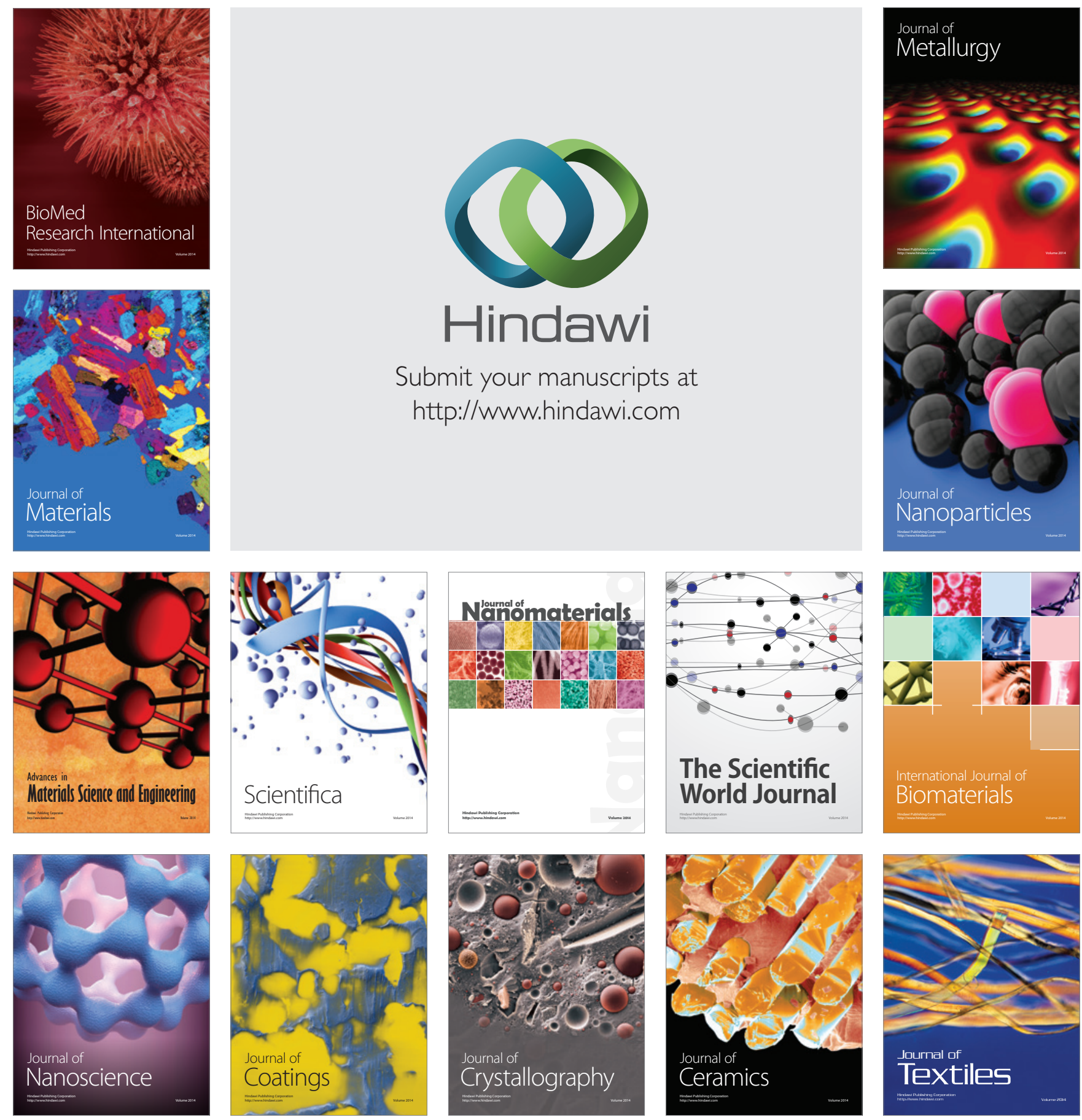